\title{
A Vectorcardiographic Evaluation of the Consensus Criteria for Early Repolarization
}

\author{
Peter L Sørensen ${ }^{1}$, Kasper Sørensen ${ }^{1}$, Jacob Melgaard ${ }^{1}$, Johannes J Struijk ${ }^{1}$, Steen M Hansen ${ }^{1}$, Jørgen \\ K Kanters ${ }^{2}$, Jonas B Nielsen ${ }^{3}$, Jesper H Svendsen ${ }^{3}$, Stig Haunsoe ${ }^{3}$, Lars Koeber ${ }^{3}$, Anders G Holst ${ }^{3}$, \\ Adrian Pietersen ${ }^{4}$, Christian Torp-Pedersen ${ }^{1}$, Freddy K Lippert ${ }^{5}$, Claus Graff ${ }^{1}$ \\ ${ }^{1}$ Aalborg University, Department of Health Science and Technology, Aalborg, Denmark \\ ${ }^{2}$ University of Copenhagen, Department of Biomedical Sciences, Copenhagen, Denmark \\ ${ }^{3}$ Rigshospitalet, Copenhagen University Hospital, Heart Centre, Department of Cardiology \\ Copenhagen, Denmark \\ ${ }^{4}$ Copenhagen General Practitioners’ Laboratory (KPLL), Copenhagen, Denmark \\ ${ }^{5}$ Emergency Medical Services, University of Copenhagen, Copenhagen, Denmark
}

\begin{abstract}
A 2015 consensus paper proposed a unified definition of the early repolarization (ER) pattern based upon quantification of end-QRS notches and slurs in the ECG. In this study we investigated the relationship between end$Q R S$ concave "bite" segments in the vectorcardiogram (VCG) and the consensus ER pattern.

We reviewed digital 12-lead ECG recordings from 1561 subjects in whom out-of-hospital cardiac arrest was later witnessed. ECGs were made available by the Copenhagen General Practitioners' Laboratory. The consensus criteria were used to quantify notches and slurs. ER manifestations were divided into 3 types: A) notches only B) notch and slur $C$ ) slurs only. An automatic algorithm developed by the authors were used to quantify bites in $3 D$.

85 of the 1561 subjects (5.4\%) fulfilled the ECG consensus criteria for ER. End-QRS bites were present in $100 \%$ of cases for types A and B and in $92 \%$ of the cases for type C. Subjects with ER type A had the highest median bite amplitude with $79 \mu V$, followed by $60 \mu V$ for type $B$ and $36 \mu V$ for type $C$.

Our investigation of end-QRS morphology in ER suggest the existence of a continuous transition from slurring to notching governed by the size of 3D VCG bites.
\end{abstract}

\section{Introduction}

Ever since Haissaguerre et al. [1] showed an association between the early repolarization (ER) pattern and sudden cardiac arrest, the definition and identification of ER has been a topic of controversy [2]. A 2015 consensus paper proposed a unified definition of ER based upon electrocardiographic (ECG) quantification of end-QRS notches and slurs [3]. The aim of this current study was to investigate the association between end-QRS notches and slurs in the ECG and concave "bite" segments in the vectorcardiogram (VCG).

\section{Methods and materials}

Digital 12-lead ECG recordings were obtained from 1561 subjects in whom out-of-hospital cardiac arrest was later witnessed. The ECGs were recorded by Copenhagen General Practitioners' Laboratory, who record and store standardized and high quality ECGs [4]. All ECGs were processed using the Marquette 12SL algorithm [5]. The Marquette 12SL algorithm generates a 12-lead median beat based upon an ECG tracing.

\subsection{Electrocardiographic quantification}

The ER ECG quantification was performed on the 12lead median beat generated by the Marquette 12SL algorithm. All 1561 ECGs were manually assessed for the presence of ER by one of the authors. Notches and slurs were quantified according to the 2015 consensus paper, see Figure 1. The amplitude of notches and slurs were measured at J peak (Jp). In accordance with the 2015 consensus paper, the presence of ER was evaluated based upon the following criteria:

1. An end-QRS notch or slur on the downslope of a prominent $\mathrm{R}$-wave. If there is a notch, it should lie entirely above the baseline. The onset of a slur must also be above the baseline.

2. $\mathrm{Jp}$ is $\geq 0.1 \mathrm{mV}$ in 2 or more contiguous leads of the 12-lead ECG, excluding leads $\mathrm{V}_{1}$ to $\mathrm{V}_{3}$.

3. QRS duration is $<120 \mathrm{~ms}$ 


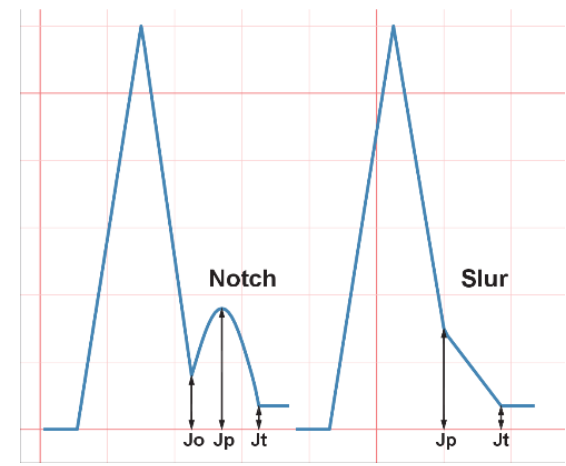

Figure 1. Illustration of a notch and a slur and the denotations suggested in the 2015 consensus paper. J onset (Jo) denotes the onset of a notch. J peak (Jp) denotes the peak of a notch or the onset of a slur. $\mathrm{J}$ termination (Jt) denote the end of a notch or slur.

For a human observer, small notches are difficult to distinguish from slurs, hence we applied a threshold of 10 $\mu \mathrm{V}$ between Jo and Jp for a notch to be coded. ER manifestations were divided into 3 types: A) notches only B) notch and slur C) slurs only.

\subsection{Vectorcardiographic quantification}

3D VCGs were derived from the 12-lead median beat using the inverse Dower transform [6]. As illustrated in Figure 2, a VCG bite can be defined as a section of the loop which rotates in the opposite direction of the main part of the loop [7]. We defined an end-QRS bite as having an onset within the terminal half of the loop and with an offset within the last quarter of the loop.

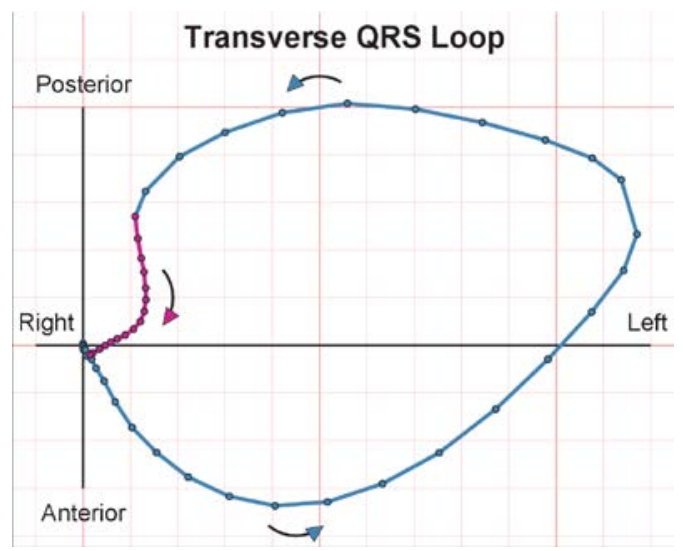

Figure 2. An illustration of a transverse QRS loop with an endQRS bite. Notice how the first part of the loop (in blue) has a counterclockwise rotation, while the terminal part of the loop (in magenta) has a clockwise rotation.

For this study we developed an automatic algorithm for delineating 3D bites. The algorithm is based upon two earlier works in the field: an automatic algorithm for delineation of 2D bites described by Edenbrandt et al. [7] and a paper by Morikawa et al. [8] where the QRS loop was incrementally rotated in order to manually identify the largest possible bite area.

The algorithm for this current study consists of the following steps: The 3D QRS loop is rotated in $10^{\circ}$ increments from $-90^{\circ}$ to $90^{\circ}$ in both the sagittal and frontal planes, this equates to 361 rotations, see Figure 3. For each rotation, the 3D QRS loop is projected onto the transverse plane and the 2D bite delineation algorithm from Edenbrandt et al. is applied. As illustrated in Figure 4, each delineated bite is quantified by amplitude, duration and area. Projections that include figure-of-eights are excluded. A minimum threshold of $10 \mu \mathrm{V}$ is applied for end-QRS bites. Following the 361 rotations, bites with more than a two sample overlap are combined. In the final step of the algorithm, the bite with largest amplitude is selected.

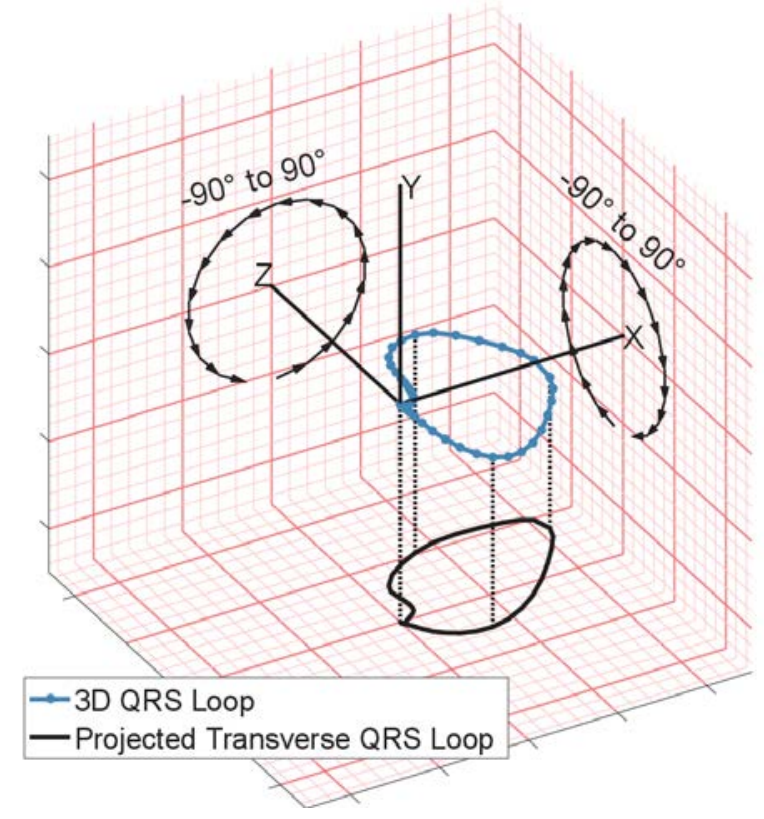

Figure 3. The 3D QRS loop is rotated $\pm 90^{\circ}$ in both the sagittal and frontal plane resulting in 361 rotations. Each rotation is projected onto the transverse plane as illustrated.

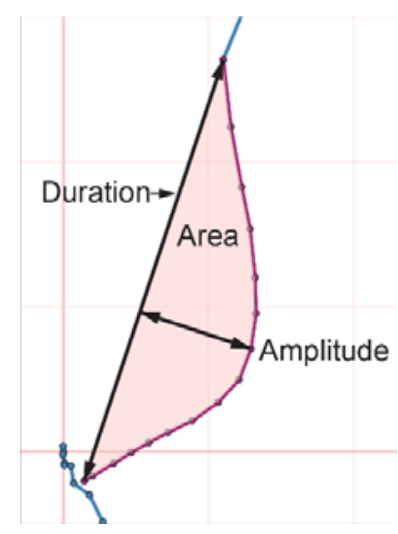

Figure 4. A VCG bite can be quantified by its duration, area and amplitude. 


\section{Results}

85 (5.4\%) of the 1561 subjects met the 2015 consensus criteria for ER. Table 1 shows the distribution of end-QRS morphology, 15 subjects had notches only (type A), 34 had both notches and slurs (type B) and 36 subjects had slurs only (type c). End-QRS bites were present in $100 \%$ of cases for types A and B, and in $92 \%$ of the cases for type C.

Table 1. End-QRS Morphology in ECG and VCG.

\begin{tabular}{llll}
\hline ER Type & $\begin{array}{l}\text { End-QRS } \\
\text { Morphology }\end{array}$ & $\begin{array}{l}\text { Number } \\
\text { of } \\
\text { Subjects }\end{array}$ & $\begin{array}{l}\text { \% of cases } \\
\text { with } \\
\text { QRS bite }\end{array}$ \\
\hline A & Notches only & 15 & $100 \%$ \\
B & Notch and slur & 34 & $100 \%$ \\
C & Slurs only & 36 & $92 \%$ \\
\hline
\end{tabular}

Bite amplitude distributed by end-QRS morphology in the ECG is shown in Figure 5. Bite amplitude was measured in the projected plane which resulted in the largest bite amplitude. Subjects with ER type A had the highest median bite amplitude with $79 \mu \mathrm{V}$, followed by 60 $\mu \mathrm{V}$ for type $\mathrm{B}$ and $36 \mu \mathrm{V}$ for type $\mathrm{C}$.

Figures $6 \mathrm{a}$ and $6 \mathrm{~b}$ illustrate the usefulness of the developed 3D bite delineating algorithm. Using the 3 standard viewing planes in Figure 6a the bite amplitude is estimated to be $33 \mu \mathrm{V}$. However, as shown in Figure 6b, $3 \mathrm{D}$ rotation of the loop unveils a larger bite amplitude of $113 \mu \mathrm{V}$.

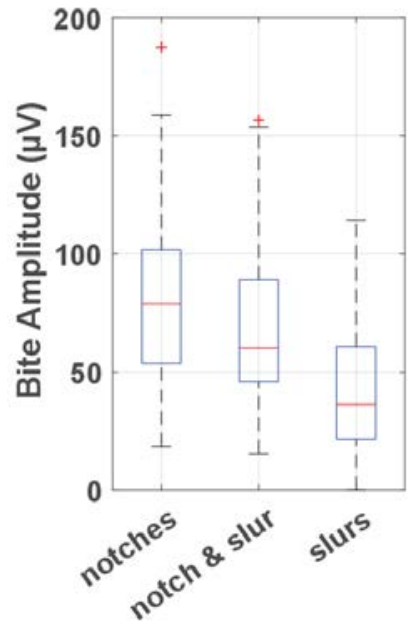

Figure 5. End-QRS bite amplitude grouped by end-QRS ECG morphology.

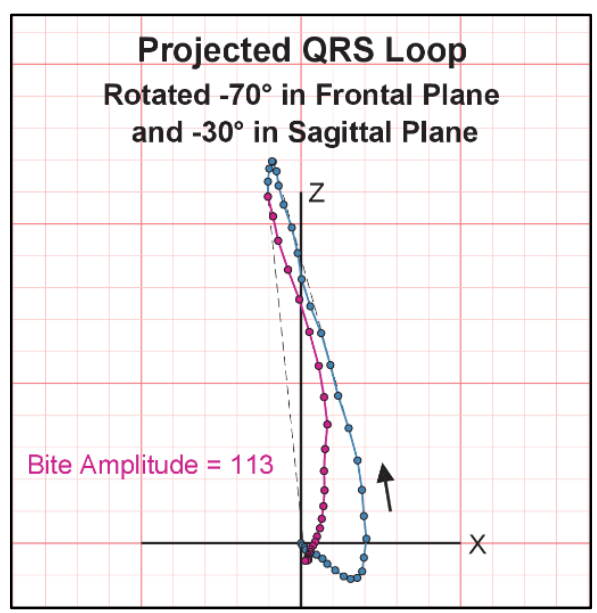

Figure 6b. Illustrates how a 3D rotation can reveal a larger bite than what is visible in the 3 standard planes.
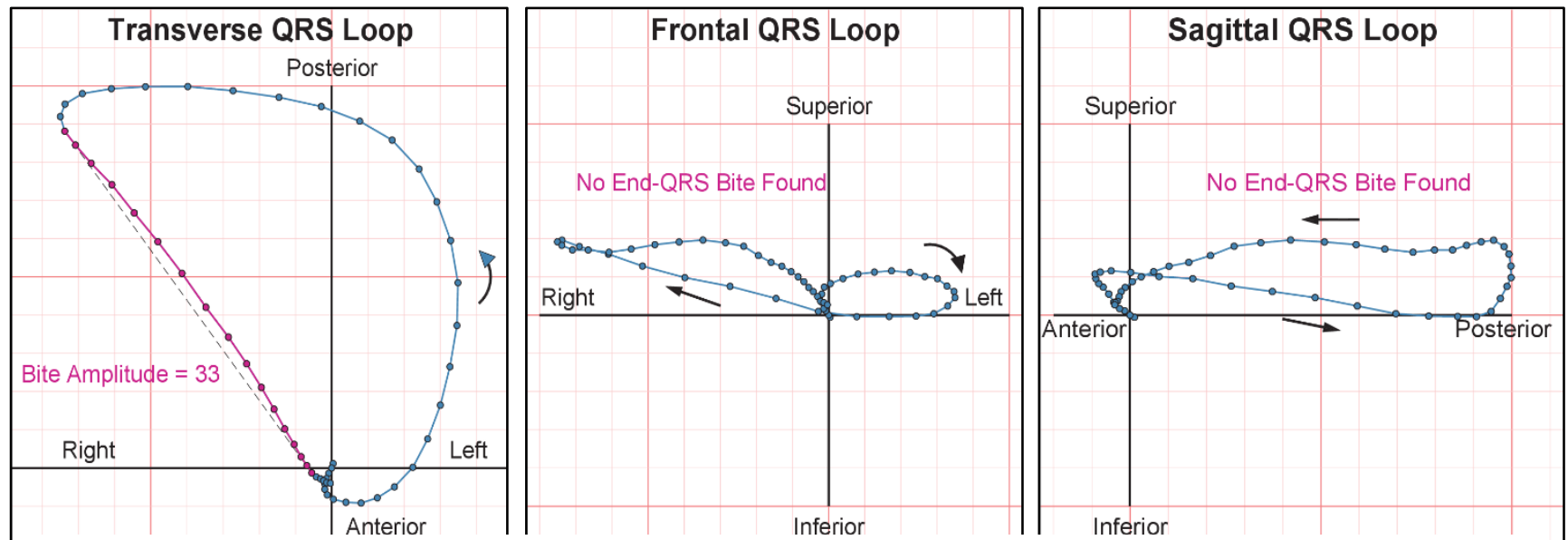

Figure 6a. Example of transverse, frontal and sagittal QRS loops for a single subject. Only in the transverse plane is a relatively small bite visible. 


\section{Discussion}

Our investigation of end-QRS morphology in ER suggests the existence of a continuous transition from slurring to notching governed by the size of 3D VCG bites. This novel quantification of ER is interesting as it provides a new tool for differentiating within the ER pattern. In the 10 years since Haissaguerre et al. [1] first showed the association between ER and sudden cardiac arrest, the search has been ongoing for a way to differentiate between the "malignant" and the "benign" ER pattern [9]. A recent meta-analysis by $\mathrm{Wu}$ et al. [10] found that an ER morphology of end-QRS notching was associated with an increased risk of arrhythmic death. From a VCG point of view this would make sense, as we have observed that notches are associated with relatively larger bites than slurs i.e. a larger alteration of the electrical signal in the terminal part of the QRS loop. We are planning to investigate the relationship between end-QRS bites and cardiac risk in the future.

A possible limitation of the developed algorithm is the definition of the search region. We observed a few cases where it was difficult to discern whether a bite should be categorized as an end-QRS bite or a mid-QRS bite. This issue is inherently linked to the "fragmentation or ER" conundrum described in the 2015 consensus paper. If the search region is too narrow the algorithm might miss bites associated with ER, while if the search region is too wide it will include bites associated with fragmentation.

\section{References}

[1] Michel Haïssaguerre, M.D., Nicolas Derval, M.D., Frederic Sacher MD, Laurence Jesel, M.D., Isabel Deisenhofer, M.D., Luc de Roy MD, Jean-Luc Pasquié, M.D., Ph.D., Akihiko Nogami, M.D., Dominique Babuty MD, et al. Sudden Cardiac Arrest Associated with Early Repolarization. T h e new engl journa l of Med 2008; 358: 2016-2023.

[2] Heng SJ, Clark EN, MacFarlane PW. End QRS notching or slurring in the electrocardiogram: Influence on the definition of 'early repolarization'. J Am Coll Cardiol 2012; 60: 947-948.
[3] MacFarlane PW, Antzelevitch C, Haissaguerre M, et al. The early repolarization pattern: A consensus paper. $J$ Am Coll Cardiol 2015; 66: 470-477.

[4] Rasmussen PV, Nielsen JB, Pietersen A, et al. Electrocardiographic Precordial ST-Segment Deviations and the Risk of Cardiovascular Death: Results From the Copenhagen ECG Study. J Am Heart Assoc 2014; 3: e000549.

[5] Healthcare G. Marquette ${ }^{\mathrm{TM}}$ 12SL ${ }^{\mathrm{TM}}$ ECG Analysis Program Physician's Guidehttp://www3.gehealthcare.com/en/support/suppor t_documentation_library (2015).

[6] Edenbrandt L, Pahlm O. Vectorcardiogram synthesized from a 12-lead ECG: Superiority of the inverse Dower matrix. J Electrocardiol 1988; 21: 361-367.

[7] Edenbrandt L, Ek A, Lundh B, et al. Vectorcardiographic bites. A method for detection and quantification applied on a normal material. $J$ Electrocardiol 1989; 22: 325-331.

[8] Morikawa J, Kitamura K, Habuchi Y, et al. Threedimensional vectorcardiography (3-D VCG) by computer graphics in old myocardial infarction. Angiology 1987; 38: 449-456.

[9] Tikkanen JT, Huikuri H V. Characteristics of 'malignant' vs. 'benign' electrocardiographic patterns of early repolarization. J Electrocardiol 2015; 48: 390 394.

[10] Wu SH, Lin XX, Cheng YJ, et al. Early repolarization pattern and risk for arrhythmia death: A meta-analysis. J Am Coll Cardiol 2013; 61: 645-650.

Address for correspondence.

Peter Lyngø Sørensen

Aalborg University

Faculty of Medicine

Department of Health Science and Technology

Laboratory for Cardiotechnology

Fredrik Bajers Vej 7 C2-203

9220 Aalborg, Denmark

pls@hst.aau.dk 